\title{
Kinematical Structure of the Local Interstellar Medium
}

\author{
R. Génova, J. E. Beckman, and J. Rodríguez Álamo
}

Instituto de Astrofísica de Canarias, Vía Láctea s/n, E-38200 La Laguna, Tenerife, Spain

Abstract. Observations of interstellar $\mathrm{Na}$ I in the spectra of 93 stars within 315 pc from the Sun show that it lies in a tunnel of gas moving away from ScorpioCentaurus and is surrounded by gas moving toward the Galactic center.

Gas approaches the Sun from Scorpio-Centaurus expanding from $(r, l, b)=(160$ pc, $313^{\circ} 7,+28^{\circ} 2$ ) with LSR velocity $15.3 \mathrm{~km} \mathrm{~s}^{-1}$. The radius of this shell is 153 pc.

We identify these clouds:

$D$ : velocity vector $\left(v_{\mathrm{d}}, l_{\mathrm{d}}, b_{\mathrm{d}}\right)=\left(+7.2 \mathrm{~km} \mathrm{~s}^{-1}, 305^{\circ} 1,-13^{\circ} 5\right)$, above and below the Galactic plane (GP) in the range of Galactic longitudes $357^{\circ}-55^{\circ}$.

$C$ : velocity vector $\left(v_{c}, l_{c}, b_{c}\right)=\left(+11.5 \mathrm{~km} \mathrm{~s}^{-1}, 349^{\circ} 0,-35^{\circ} 2\right)$, above and below the GP in the range $30^{\circ} \leq l \leq 110^{\circ}$.

$M$ : velocity vector $\left(v_{\mathrm{m}}, l_{\mathrm{m}}, b_{\mathrm{m}}\right)=\left(+21.9 \mathrm{~km} \mathrm{~s}^{-1}, 34^{\circ} 2,+1^{\circ} 5\right)$, above and below the GP in the range $100^{\circ} \leq l \leq 130^{\circ}$.

$P$ : velocity vector $\left(v_{\mathrm{p}}, l_{\mathrm{p}}, b_{\mathrm{p}}\right)=\left(+13.8 \mathrm{~km} \mathrm{~s}^{-1}, 224^{\circ} 9,+5^{\circ} 4\right)$, above and below the GP from $l \sim 120^{\circ}$ to the limit of our data at $l \sim 210^{\circ}$.

$E$ : velocity vector $\left(v_{\mathrm{e}}, l_{\mathrm{e}}, b_{\mathrm{e}}\right)=\left(+16.8 \mathrm{~km} \mathrm{~s}^{-1}, 208^{\circ} 4,+6^{\circ} 2\right)$ in the range $160^{\circ} \leq l$ $\leq 185^{\circ}$ and $-10^{\circ} \leq b \leq-35^{\circ}$.

$A$ : velocity vector $\left(v_{\mathrm{a}}, l_{\mathrm{a}}, b_{\mathrm{a}}\right)=\left(+12.9 \mathrm{~km} \mathrm{~s}^{-1}, 73^{\circ} 6,-5^{\circ} 6\right)$ towards the Galactic anti-center, below the GP.

$I$ : velocity vector $\left(v_{\mathrm{i}}, l_{\mathrm{i}}, b_{\mathrm{i}}\right)=\left(+37.7 \mathrm{~km} \mathrm{~s}^{-1}, 132^{\circ} 8,-64^{\circ} 3\right)$ towards the Galactic anti-center, above the GP.

\section{Historical Perspective}

The X-ray emission ubiquitously observed in the Local Interstellar Medium (LISM) (Sanders et al. 1977) shows that the Sun and the nearest stars are surrounded by gas with the characteristics typical of the interiors of interstellar bubbles produced by supernova remnants and stellar winds, suggesting that the Sun is within a large interstellar bubble (Cox, \& Reynolds 1987), consequently named "Local Bubble" (LB), whose detailed origin is still unknown. The interior of this LB is being modified by the effect of stellar winds and supernova explosions produced by stars that lie within the LB and, as new instrumental developments allow a deeper study of the shortest lines of sight, the complexity of the kinematics and spatial distribution of the local gas is becoming evident. This gas is moving away from a region of the sky occupied by the Scorpio-Centaurus OB association and its associated subgroups and it 
was naturally suggested that the supernova explosions and stellar winds produced by its most massive stars were the engines that set the gas in motion (Bertaux, \& Blamont 1971; Adams, \& Frisch 1977).

The first comprehensive attempt to explore the overall spatial distribution of the local gas closest to the Sun was made by Génova et al. (1990). These authors showed the presence of an asymmetry in the distribution of the local interstellar gas with high densities observed in the hemisphere that contains the Galactic center and low densities in the opposite Galactic hemisphere. The large-scale motion of the local gas toward the Galactic anti-center hemisphere previously observed by Crutcher (1982) was also detected in these low resolution data. When high-resolution optical data of the shortest lines of sight became available a new vision of the LISM kinematics emerged, showing that the local kinematical structure is not as homogeneous as previously thought. Several cloudlets were detected in the immediate vicinity of the Sun (Lallement, Vidal-Madjar, \& Ferlet 1986; Lallement \& Bertin 1992), nevertheless these cloudlets share a common motion toward the Galactic anti-centre hemisphere. The Sun happens to be submerged in one of the cloudlets of this ensemble, the Local Cloud (LC). The presence of this cloud was inferred from the early 70's through the detection of $\mathrm{H}$ and $\mathrm{He}$ atoms of interstellar origin flowing in the inner heliosphere (Bertaux, \& Blamont 1971; Adams, \& Frisch 1977) and it has now been detected in the spectra of the nearest stars (Lallement et al. 1994).

\section{Recent Results}

The presence of spheroidal shells approaching the Sun from the ScorpioCentaurus association has been shown by Crawford (1991) and Génova et al. (1997a). The shell detected by Crawford (1991) is centered at $(l, b) \sim\left(320^{\circ}\right.$, $+10^{\circ}$ ) at $140 \pm 20 \mathrm{pc}$ from the Sun, its radius is $110 \pm 10 \mathrm{pc}$, it is expanding with velocity $7 \mathrm{~km} \mathrm{~s}^{-1}$. The shell detected by Génova et al. (1997a) is faster, its expansion velocity being $15.3 \mathrm{~km} \mathrm{~s}^{-1}$, and is centered at $\sim 160 \mathrm{pc}$ from the Sun at $(l, b) \sim\left(313^{\circ},+28^{\circ}\right)$, its radius is $\sim 153 \mathrm{pc}$. These values place the centers of these shells well within the Scorpio-Centaurus association. Even if they may have a common direction of origin, the difference in their expansion velocities suggests that these shells may not be related, most probably they are shell-like filaments emitted from Scorpio-Centaurus at different epochs. In particular, the dimensions of the shell detected by Génova et al. (1997a) place its edge very close to the Sun in direction toward Scorpio-Centaurus, also its expansion velocity and the velocities of the local ensemble of clouds are of the same order; these facts suggest that the LC and its accompanying cloudlets are the leading edge of and expanding interstellar bubble that is engulfing the Sun.

A somewhat surprising result has been the recent detection of nearby interstellar clouds that show motions toward the Galactic center hemisphere. 
Interstellar $\mathrm{Na}$ I absorptions detected in the spectra of 93 stars within 315 pc from the Sun observed from the $4.2 \mathrm{~m}$ William Herschel Telescope on La Palma with a velocity uncertainty of order $1.0-1.5 \mathrm{~km} \mathrm{~s}^{-1}$ have shown the presence of the expanding shell from Scorpio-Centaurus described above as well as the 7 interstellar clouds whose velocity vectors and location in the sky are cited in the abstract (Génova et al. 1997a; Génova et al. 1997b). Four of these clouds - $A, M, C$ and $D$ - show motions toward the Galactic center hemisphere, which is in contradiction with the current ideas about the local gas kinematics. Three clouds - $P, I$, and $E-$ show motions toward the Galactic anticenter hemisphere. Cloud $P$ covers the largest area in the sky among these clouds, which seems to indicate that it lies close to the Sun. It also shows a velocity vector similar to the vector found by Crutcher (1982) in the nearby gas. Cloud $E$ is kinematically similar to $P$, both clouds are seen simultaneously in several lines of sight toward the Pleiades, these are the clouds that give rise to the kinematical structure discussed by Gordon \& Arny (1984) in the vicinity of this cluster.

\section{The Emerging Picture of the Local Gas Kinematics}

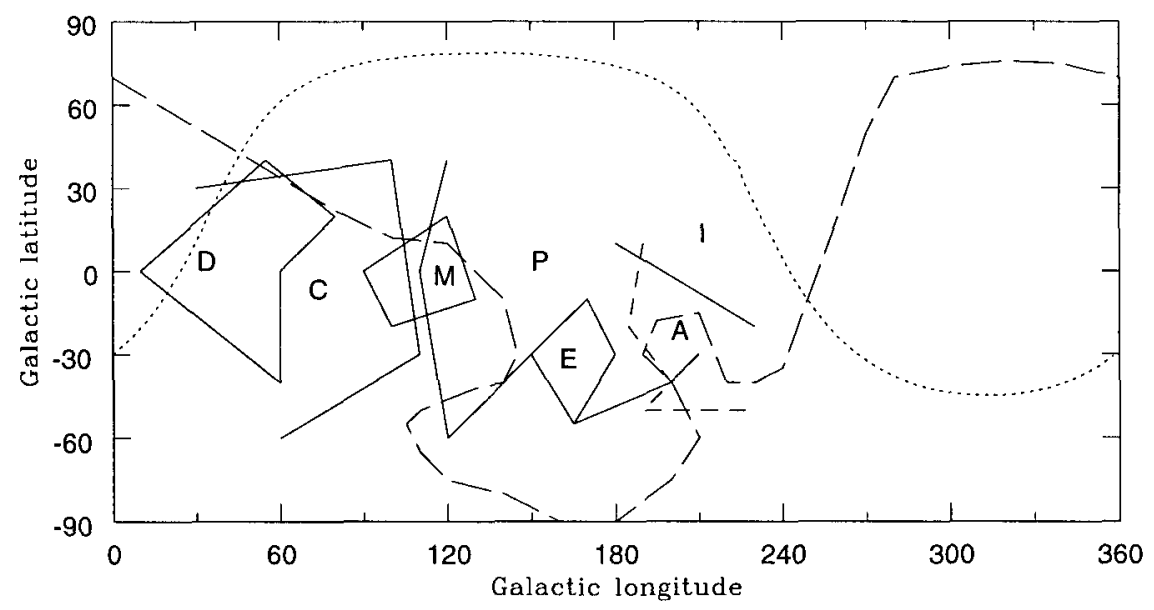

Fig. 1. The long-dashed line shows the limit between the low and high Mg II density regions identified by Génova et al. (1990) toward stars within the nearest $30 \mathrm{pc}$. The projection on the sky of the expanding shell identified by Génova et al. (1997a) is shown by the dotted line. Solid lines sketch the projections on the sky of the clouds detected close to the Sun by Génova et al. (1997a) and Génova et al. (1997b). 
As shown in Figure 1 clouds $P, I$, and $E$ are observed against the low $\mathrm{Mg}$ II density region. On the other hand, the clouds that show motions toward the Galactic center hemisphere are observed against the high Mg II density region. This morphology can be explained by means of the presence of two interstellar bubbles in the immediate vicinity of the Sun: one bubble is approaching the Sun from Scorpio-Centaurus, the nearby interstellar gas is being swept up and accelerated by the leading edge of this bubble. In the direction of the Galactic center we observe high densities produced by interstellar gas that has already accumulated in the shell of this bubble. In the opposite direction we observe a low density region toward which the former bubble is advancing. This region is in fact the interior of an older bubble whose shell is seen in the clouds that are moving toward the Galactic center hemisphere. Most absorptions in the direction of the Galactic anticenter are caused by gas very close to the Sun that has been accelerated by the ScorpioCentaurus bubble and has already passed the Sun.

Although we give precise values of the velocity vectors of these clouds we feel that given the range of distances to the background stars used to determine those velocity vectors they represent average motions of the gas, nevertheless the existence of regions where the IS gas flows towards the Galactic center hemisphere is clearly shown in our data. Further observations, in particular precise determinations of the distances to the nearest edges of these clouds, are needed to confirm this view.

\section{References}

Adams T.F., Frisch P.C. (1977): ApJ 212, 300

Bertaux J.L., Blamont J.E. (1971): A\&A 11, 200

Cox D.P., Reynolds R.J. (1987): ARA\&A 25, 303

Crawford I.A. (1991): A\&A 247, 183

Crutcher R.M. (1982): ApJ 254, 82

de Geus E.J. (1992): A\&A 262, 258

Génova R., Molaro P., Vladilo G., Beckman J.E. (1990): ApJ 355, 150

Génova R., Beckman J.E., Bowyer S., Spicer T. (1997a): ApJ 484, (page not yet assigned)

Génova R., Beckman J.E., Rodríguez Álamo J. (1997b): ApJ submitted

Gordon K.J., Arny T.T. (1984): AJ 89, 672

Lallement R., Bertin P., Ferlet R., Vidal-Madjar A., Bertaux J.L. (1994): A\&A 286, 898

Sanders W.T., Kraushaar W.L., Nousek J.A., Fried P.M. (1977): ApJL 217, L87 